\title{
Suizidbeihilfe in der Schweiz
}

\author{
M. Sc. Anita KOVACEVIC ${ }^{1}$, Dr. med. Christine BARTSCH²
}

Universität Zürich ${ }^{3}$

\begin{abstract}
Direkte aktive Sterbehilfe, auch Euthanasie genannt, bezeichnet die von einem Patienten gewünschte Tötung zur Verkürzung seiner Leidensdauer und ist in den meisten Ländern der Erde gesetzlich verboten, so auch in der Schweiz. Die durch Artikel 115 des Strafgesetzbuchs (StGB) legitimierte Beihilfe zum Suizid ist demgegenüber ein Vorgang, bei dem die sterbewillige Person durch die Bereitstellung einer tödlichen Medikamentendosis, die sie selbständig aufnehmen muss, von Dritten unterstützt wird. Diese Assistenz muss allerdings frei von selbstsüchtigen Beweggründen geschehen. Jährlich sterben in der Schweiz etwa 700 Menschen nach assistiertem Suizid (AS), und ihre Anzahl nimmt stetig zu. Mittels einer retrospektiven Forschungsstudie an Akten aller in den Schweizerischen Instituten für Rechtsmedizin (IRM) archivierten AS-Fälle aus mehr als 30 Jahren wurden Daten zu insgesamt 3.666 Todesfällen erfasst und analysiert. Im Beitrag werden erste Ergebnisse sowie festgestellte Problemkonstellationen zur Veranschaulichung der Situation präsentiert.
\end{abstract}

Keywords: Suizidbeihilfeorganisationen, Sterbehilfe, Suizidbeihilfe, Natriumpentobarbital (NaP), NFP 67 Lebensende

\section{Einleitung}

Der Umgang mit Sterben und Tod scheint sich über die Zeit stark zu verändern. Wo früher noch von einem selbstverständlichen Sterben gesprochen wurde, steht heute ein kontrolliertes

\footnotetext{
${ }^{1}$ Anita Kovacevic, M. Sc. in Psychologie, Institut für forensische Psychiatrie und Psychotherapie, IFPP, Wiesenstrasse 39, Langenthal, anita-kovacevic@gmx.ch. Kovacevic ist Psychologin am IFPP in Langenthal. Sie arbeitete als wissenschaftliche Hilfskraft und wissenschaftliche Mitarbeiterin im Projekt Der assistierte Suizid-Entwicklungen währen der letzten 30 Jahre.

${ }^{2}$ Prof. Dr. Christine Bartsch ist Rechtsmedizinerin und leitete zuletzt die Abteilung für Forensische Medizin und Bildgebung am Institut für Rechtsmedizin der Universität Zürich. Dazu war sie Dozentin. Inzwischen arbeitet sie im deutschen Bremerhaven in einem Institut für Polar- und Meeresforschung. Das Projekt Der assistierte Suizid-Entwicklungen während der letzten 30 Jahre leitete sie hauptverantwortlich.

${ }^{3}$ Das Projekt Der assistierte Suizid - Entwicklungen während der letzten 30 Jahre des Nationalen Forschungsprogramms (NFP 67) Lebensende ist ein interdisziplinär und multizentrisch angelegtes Gemeinschaftsprojekt, von Prof. Dr. Bartsch hauptverantwortlich geleitet und in Kooperation mit Prof. Dr. med. Thomas Reisch (Chefarzt der Klinik für Depression \& Angst / Ärztlicher Direktor des Psychiatriezentrums Münsingen) und PD Dr. phil. Vladeta Ajdacic (Sozialwissenschaftler und Epidemiologe an der Klinik für Psychiatrie, Psychotherapie und Psychosomatik der Universität Zürich) durchgeführt. Im Gesamtkonzept waren 17 Projektmitarbeitende tätig.
} 
Sterben im Vordergrund. Vielfach ist die Rede von einem ärztlich gesteuerten und kontrollierten Prozess (Schwarzenegger et al. 2010). Ergebnisse einer europäischen Studie aus dem Jahr 2003 haben dies u.a. für die Schweiz eindrücklich gezeigt (van der Heide et al. 2003): Von den untersuchten Todesfällen geschahen nur etwa die Hälfte plötzlich oder ohne ärztliche Kontrolle. In allen anderen Fällen kam es zu ärztlichen Entscheidungen am Lebensende.

Das Lebensende wird zu einer individuellen, letzten Planungsaufgabe, um die man sich gut kümmern möchte, damit das Ende möglichst kontrolliert und selbstbestimmt gestaltet werden kann. Nichts soll mehr dem Zufall überlassen werden. Ein friedliches, schmerzfreies und würdevolles Sterben wird angestrebt, auch wenn dies bedeutet, dass man Dritte dabei in die Pflicht nehmen muss. Man möchte Hilfe beim resp. zum Sterben erhalten (Mathwig 2010). In diesem Zusammenhang spricht man oft von Sterbehilfe und Suizidbeihilfe. In der Schweiz ist aktive Sterbehilfe im Artikel 114 des Strafgesetzbuchs (StGB) geregelt und verbietet die Tötung auf Verlangen. Die Suizidbeihilfe, auch assistierter Suizid (AS) genannt, ist dagegen unter bestimmten Voraussetzungen erlaubt, wie dies in Artikel 115 StGB formuliert wurde. Noch weitere rechtliche und standesethische Vorgaben bestehen im Kontext des AS, die das Prozedere an unterschiedlichen Stellen regulieren. Mittlerweile gibt es sechs Suizidbeihilfeorganisationen, die als Vereine anbieten, Suizidwilligen beim selbstbestimmten und würdevollen Sterben $\mathrm{zu}$ assistieren.

Das Bundesamt für Statistik (BfS) hat im Jahr 2009 erstmals Zahlen zur Suizidbeihilfe in der Schweiz veröffentlicht. Bis in das Jahr 1997 wurden alle diese Fälle als Suizide durch Vergiftung klassifiziert. Erst seit 1998 werden AS in den Totenscheinen der Verstorbenen speziell gekennzeichnet und statistisch separat erfasst. Die neuesten Zahlen des BfS zeigen für das Jahr 2014, dass 742 Menschen mit Wohnsitz in der Schweiz mit Hilfe eines AS aus dem Leben geschieden sind, das ist ein Viertel mehr als im Vorjahr. Darunter waren 422 Frauen und 320 Männer. Die Verstorbenen waren zu 94 Prozent über 55-jährig und litten in den allermeisten Fällen unter einer schwerwiegenden, terminalen Krankheit (BfS 2016).

Anders als beispielsweise in Belgien, den Niederlanden oder dem US-amerikanischen Bundesstaat Oregon existiert in der Schweiz keine zentrale Meldestelle für assistierte Suizide. Das bedeutet, dass bis heute keine gesamtschweizerischen Daten zur Suizidbeihilfe verfügbar sind (Andorno 2013). In der Schweiz wird ein nicht natürlicher Todesfall wie der AS als aussergewöhnlicher Todesfall (agT) definiert und fällt somit unter Artikel 253 der Strafprozessordnung (SR 321.0 StPO). In diesen Fällen wird eine amtsärztliche Leichenschau, eine sogenannte Legalinspektion, durch Rechtsmedizinerinnen und Rechtsmediziner oder speziell ausgebildete Amtsärzte durchgeführt. Nach einer rechtsmedizinischen Untersuchung werden die Unterlagen zu diesen Fällen als Kopien in den Archiven der Institute für Rechtsmedizin (IRM) aufbewahrt. Die Frage, ob wirklich jeder AS als agT gemeldet und untersucht wird, muss mit Nein beantwortet werden, da wir von einem zufällig nachgemeldeten Fall Kenntnis erlangt haben und hierdurch feststellen konnten, dass eine Nichtmeldung nicht zwingend erkannt werden muss.

Um dennoch einen Überblick über die Entwicklung des AS in der Schweiz inklusive der Daten zum sogenannten Suizidtourismus aus den letzten mehr als 30 Jahren zu erhalten und das Phänomen damit besser verstehen und interpretieren zu können, wurde 2012 ein insgesamt vierjähriges Forschungsprojekt $\mathrm{zu}$ diesem Thema mit in das grosse Forschungspro- 
gramm des Schweizerischen Nationalfonds NFP 67 Lebensende aufgenommen. Hierzu wurden Daten aus den archivierten Akten der damals sieben Schweizerischen Institute für Rechtsmedizin (IRM) retrospektiv erfasst und ausgewertet.

Im vorliegenden Beitrag wird die Situation des AS in der Schweiz anhand erster Studienergebnisse aufgezeigt und unter Berücksichtigung der existierenden Regulierungen diskutiert. Hierzu wird das Forschungsprojekt detailliert vorgestellt, das die AS-Praxis aus rechtsmedizinischer Perspektive beleuchtet.

\section{Rechtliche Grundlagen der Sterbehilfe und des assistierten Suizids in der Schweiz}

Die rechtlichen Regelungen der Sterbe- und Suizidbeihilfe werden nicht nur in der Schweiz kontrovers diskutiert, sondern auch in anderen europäischen Ländern, nachweislich sogar weltweit. In der Schweiz gab es in der Vergangenheit immer wieder politische Initiativen, die forderten, an der Situation und der rechtlichen Regelung etwas zu verändern. Die Ergebnisse des genannten Forschungsprojekts werden mit Spannung erwartet und bieten für die weitere Diskussion eine wissenschaftlich fundierte Grundlage.

Sterbehilfe und Suizidbeihilfe unterscheiden sich nicht nur begrifflich, sondern beinhalten unterschiedliche Vorgänge. Bei der direkten aktiven Sterbehilfe, in gewissen Ländern und Sprachen wird der Begriff synonym mit Euthanasie verwendet, wird damit eine gezielte Tötung auf Verlangen bezeichnet, die das Leiden eines erkrankten Menschen verkürzen soll. Die aktive Sterbehilfe beinhaltet die Handlung eines Mitmenschen (meist das Verabreichen eines Medikaments), welche über kurze oder lange Frist zum Tode des Patienten führt (Schwarzenegger et al. 2010). In der Schweiz ist die aktive Sterbehilfe im Strafgesetzbuch (StGB) durch die Artikel 111 (vorsätzliche Tötung), 113 (Totschlag) und 114 (Tötung auf Verlangen) unter Strafe gestellt.

Unter Beihilfe zum Suizid bzw. assistiertem Suizid wird der Sachverhalt verstanden, dass die betroffene Person (Suizidwillige/r) bei ihrem Vorhaben (Suizid) unterstützt wird, nämlich bei der Beschaffung des tödlich wirkenden Medikaments (zumeist das Betäubungsmittel Natriumpentobarbital (NaP)) (Schwarzenegger et al. 2010). Dieses Medikament muss von der Person selbständig ein- bzw. aufgenommen werden (oral, intravenös oder per Magensonde). Somit muss die letzte, den Tod herbeiführende Handlung von der betroffenen Person eigenhändig ausgeführt werden, in der juristischen Sprache wird auch von der Tatherrschaft über den todbringenden Akt gesprochen. Neben der Tatherrschaft ist für die strafrechtliche Beurteilung der Suizidbeihilfe massgebend, dass die/der Suizidwillige den Suizidwunsch frei und überlegt gewählt hat und zu diesem Zeitpunkt urteilsfähig war. Seitens der assistierenden Personen dürfen nach Art. 115 StGB des Weiteren keine selbstsüchtigen Beweggründe vorliegen, damit die Suizidbeihilfe straffrei bleibt (Brunner 2010).

Die unabdingbare Voraussetzung für die Ausführung von AS ist die Urteilsfähigkeit, deren Vorhandensein laut Richtlinien Zur Betreuung von Patientinnen und Patienten am Lebensende der Schweizerischen Akademie der Medizinischen Wissenschaften (SAMW) im Vorfeld der Handlung festgestellt werden muss (SAMW 2013). Nur wer urteilsfähig ist, kann sich eigenverantwortlich für die Beendigung seines Lebens entscheiden. Die Urteilsfähigkeit ergibt sich aus Artikel 16 des Schweizerischen Zivilgesetzbuchs (ZGB). Nach diesem Artikel ist jeder ur- 
teilsfähig, dem es nicht wegen seines Kindesalters, infolge geistiger Behinderung, psychischer Störung, Rauschs oder ähnlichen Zuständen an der Fähigkeit mangelt, vernunftgemäss zu handeln. Doch bei diesen Zuständen geht man nicht per se von Urteilsunfähigkeit aus. Die Urteilsfähigkeit ist immer relativ und in einem spezifischen Kontext zu betrachten. Das bedeutet, die Beurteilung der Urteilsfähigkeit muss sich immer auf einen spezifischen Kompetenzbereich, eine spezifische Situation oder eine spezifische Entscheidung beziehen (Trachsel et al. 2015). Ist im Falle eines geplanten AS anlässlich der Überprüfung der Voraussetzungen festgestellt worden, dass an der Urteilsfähigkeit über den frei erwogenen Sterbewunsch Zweifel bestehen, darf keine Suizidbeihilfe geleistet werden. In der Schweiz gibt es bis heute keine standardisierten Richtlinien, nach denen die Urteilsfähigkeit in Bezug auf den Sterbewunsch beurteilt und dokumentiert werden kann bzw. muss.

Nach den SAMW-Richtlinien wird die Suizidbeihilfe grundsätzlich nicht als ärztliche Aufgabe angesehen. Diese räumen aber die Möglichkeit ärztlicher Suizidbeihilfe als eine Ausnahmehandlung ein, jedoch unter streng zu beachtenden Kriterien. Bei einer entsprechenden Anfrage um Suizidbeihilfe könne jede/r Ärztin/Arzt selber entscheiden, ob sie/er dies im gesetzlichen Rahmen machen möchte oder nicht. Entscheidet sich ein/e Arzt/Ärztin, Suizidbeihilfe nach den bestehenden Regelungen zu leisten, dann muss diese Entscheidung respektiert werden. Hierzu muss die Erkrankung der sterbewilligen Person in einer terminalen Phase sein. Weiter müssen alternative Möglichkeiten aufgezeigt und verworfen worden sein. Letztlich muss der Suizidwunsch wohlerwogen, andauernd und frei von äusserem Druck entstanden sein (SAMW 2013).

Aktuell gibt es sechs in der Schweiz ansässige Organisationen, die vorgeben, im Rahmen des Artikel 115 StGB ohne selbstsüchtige Beweggründe legalisierte Suizidbeihilfe zu leisten. Diese Organisationen bieten Suizidbeihilfe an, indem sie über eine/n Ärztin/Arzt das notwendige Rezept für das Suizidmittel beschaffen und der sterbewilligen Person zur Verfügung stellen. Bei Bedarf findet ein solcher Suizid in den Räumlichkeiten der jeweiligen Organisation statt (Exit 2016; Dignitas 2016).

Historisch betrachtet wurden im Jahre 1982 in der Schweiz unabhängig voneinander die ersten beiden Organisationen - EXIT Deutsche Schweiz und EXIT Association pour le Droit de Mourir dans la Dignité (A.D.M.D.) suisse romande - gegründet. Sie setzen sich für die Selbstbestimmung der Menschen bezüglich des eigenen Todes ein. Laut Angaben von EXIT Deutsche Schweiz wurde im Januar 1985 erstmals offiziell ein schwer krankes Vereinsmitglied in den Freitod begleitet. Jährlich werden in der Schweiz ca. 780 Suizidbegleitungen von in der Schweiz wohnhaften Personen durchgeführt. Für eine Suizidassistenz durch eine der Vereinigungen ist einerseits die Mitgliedschaft erforderlich und andererseits ein Wohnrecht in der Schweiz Voraussetzung. Die Suizidbegleiter arbeiten laut Angaben der Vereinigung ehrenamtlich und erhalten lediglich eine Pauschale für ihre Spesen (Exit 2016).

Eine Abspaltung des grössten Suizidbeihilfevereins EXIT Deutsche Schweiz stellt der Verein DIGNITAS - Menschenwürdig leben - Menschenwürdig sterben dar, der 1998 gegründet wurde. Der in der Schweiz ansässige Verein DIGNITAS bietet auch im Ausland wohnhaften Menschen mit unheilbaren Krankheiten, unerträglichen Schmerzen oder unzumutbaren Behinderungen aller Nationalitäten die Möglichkeit einer Suizidassistenz an (Dignitas 2016). 
Bei den übrigen Vereinen handelt es sich um Ex International, life circle der Stiftung Eternal SPIRIT und Sterbehilfe Deutschland e.V. (StHD).

\section{Forschungsprojekt im NFP 67 Lebensende: Assistierte Suizide in der Schweiz - eine detaillier- te nationale Erfassung der letzten 30 Jahre}

Das Ziel dieses Projektes war es, einen Überblick über die Situation des assistierten Suizids in der Schweiz seit der Gründung der ersten Suizidbeihilfeorganisationen $1982 \mathrm{zu}$ gewinnen. Die Ergebnisse und die daraus ableitbaren Erkenntnisse bieten schliesslich eine wissenschaftlich fundierte Grundlage für weiterführende Debatten und Entscheidungen. Nicht nur regionale, sondern auch Unterschiede zwischen den verschiedenen Suizidbeihilfeorganisationen in Bezug auf Vorgehen, schriftliche Dokumentation und Klientel sowie Beweggründe für den Entschluss zum AS werden untersucht. Zudem soll das Phänomen des Suizidtourismus, d.h. die Suizidbeihilfe für im Ausland wohnhafte Menschen, beleuchtet werden. Hier interessiert besonders, ob die Fälle aus dem Ausland ebenfalls so stark zugenommen haben, wie dies in der medialen Berichterstattung immer wieder betont wird. Ausserdem ist interessant, aus welchen Ländern sie mit welchen Erkrankungen und Hauptmotiven für den AS anreisen und ob die Gruppe sich von den Schweizerinnen und Schweizern unterscheidet.

Für die Studie wurden alle in den im Jahr 2012 existierenden sieben Schweizerischen Instituten für Rechtsmedizin (IRM) untersuchten Todesfälle nach AS anhand der dort archivierten Fallakten aus den Jahren 1982 (erster offiziell begleiteter Todesfall nach AS 1985) bis 2013 (am IRM der Universität Zürich bis einschliesslich Juni 2014) retrospektiv erfasst. Die notwendigen Daten wurden aus den vorliegenden Dokumenten anhand eines eigens für die Studie konzipierten Erhebungsbogens anonym erfasst. Der Bogen enthielt grösstenteils geschlossene Fragen zu den Aspekten: rechtsmedizinische Untersuchungsart (äussere und/oder innere Leicheninspektion), sozio-demografische Variablen (Alter, Geschlecht, Zivilstatus, Beruf etc.), Vorgehen/Ablauf des AS (Applikationsform, Dokumentenlage etc.), zugrunde liegende Erkrankungen und Motive für den AS. Ein weiterer Aspekt bei der Auswertung der AS-Fälle betrifft den Umfang der in den Akten vorhandenen schriftlichen Unterlagen.

Bisher liegen einzelne Daten zu insgesamt 3666 AS-Fällen vor, darunter sind Angaben zu 1687 sogenannten Suizidtouristen, die vom BfS nicht miterfasst werden. Der Anteil an rechtsmedizinisch untersuchten AS-Todesfällen entspricht im Forschungszeitraum etwa 44\% der vom BfS dokumentierten Fälle. Das Gesamtkollektiv setzt sich aus 2191 Frauen(59.8\%) und 1475 Männern(40.2\%) im Alter zwischen 18 und 105 Jahren zusammen. Der Median liegt bei einem Sterbealter von 73.0 Jahren (Mittelwert 71.2 Jahren).

Die Aktivitäten der verschiedenen Suizidbeihilfeorganisationen haben auf dem Gebiet des AS in der Schweiz über die letzten 30 Jahre rasch und anhaltend zugenommen (Abbildung 1). Die erste offiziell dokumentierte Suizidassistenz fand im Januar 1985 statt und wurde vom Geschäftsführer von EXIT und seiner Ehefrau bei einer suizidwilligen Person durchgeführt (Exit 2016). Zwischen 1985 und Ende der Neunziger verhält sich die Anzahl mit durchschnittlich etwa 14 Fällen pro Jahr (Range $=1-34$ ) nahezu gleichbleibend. Ab dem Jahr 2000 nehmen die Fälle plötzlich stark zu und erreichen im Jahr 2006 mit 295 einen ersten Höchstwert. Im Jahr 2007 ist die Anzahl mit 226 Fällen etwas rückläufig. Im Jahr 2006 kam es zum ersten 
wichtigen Bundesgerichtsentscheid, bei welchem die ärztliche Verantwortung stark betont wurde, was insbesondere bei der Verschreibung des tödlichen Medikaments und bei der Beurteilung der Urteilsfähigkeit zu strengeren Regelungen geführt hat (BGE 2A.48/2006 und 2A.66/2006 2006a, 2006b). Ob dieser kurzfristige Rücklauf im Jahr 2007 auf den Bundesgerichtsentscheid zurückzuführen ist, bleibt eine Vermutung. Im Jahr 2013 liegt die Anzahl von AS Fällen, die in den rechtsmedizinischen Instituten archiviert wurden, bei 432. In der ersten Hälfte des Jahres 2014 konnten aus logistischen Gründen nur noch die AS-Fälle vom IRM Zürich in die Studie einbezogen werden, was die relativ geringere Anzahl erklärt.

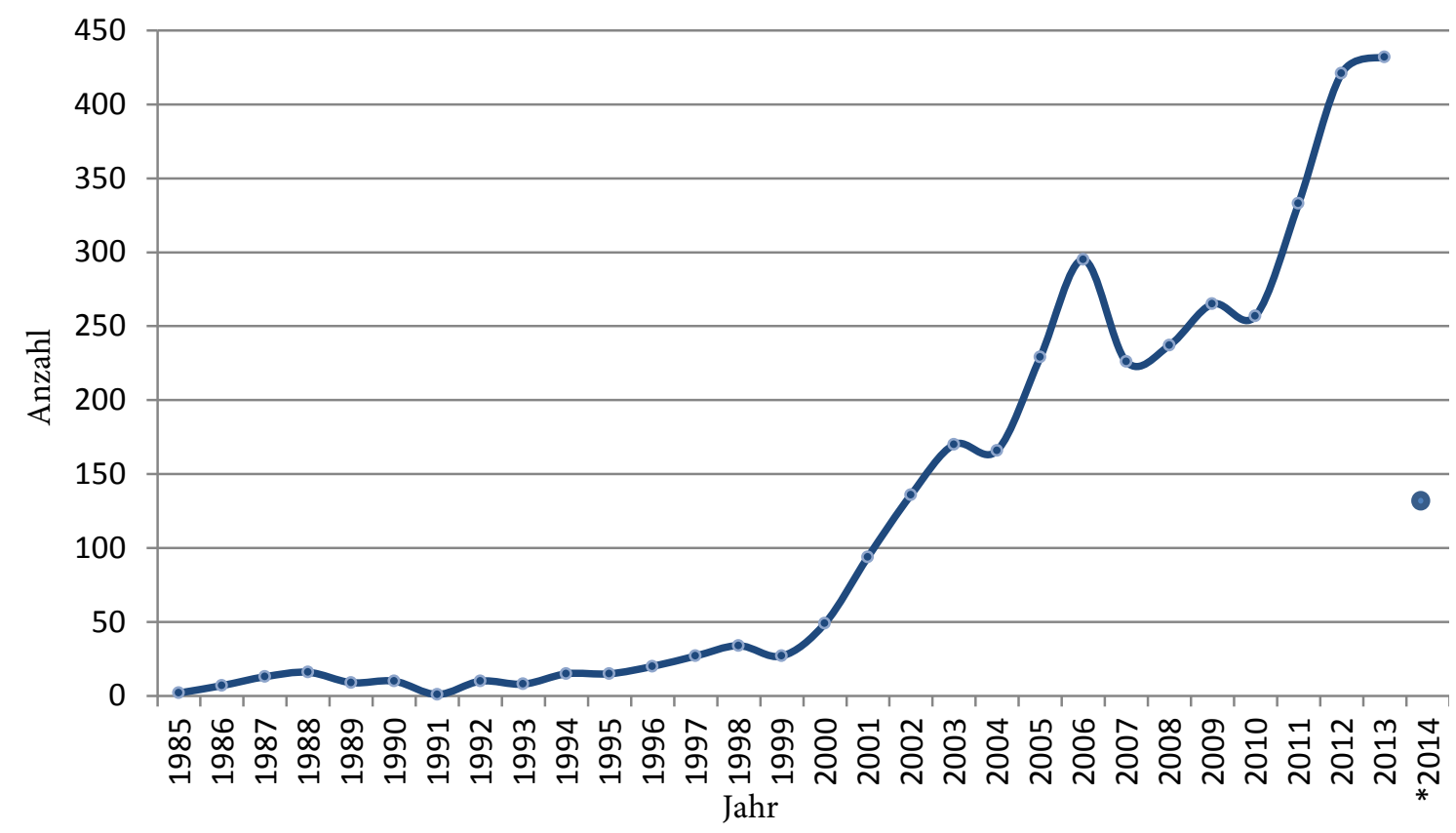

Abbildung 1: Rechtsmedizinisch archivierte Todesfälle nach assistiertem Suizid aus den Jahren 1985 bis 2014 von Personen mit Wohnort in der Schweiz und im Ausland (N=3666).

* 2014 nur 6 Monate und nur Fälle des IRM Zürich

Betrachtet man die Fallzahlen nach den einzelnen Suizidbeihilfeorganisationen, so erkennt man, dass die meisten AS-Fälle von ehemals in der Schweiz ansässigen Personen von EXITDeutsche Schweiz begleitet wurden, gefolgt von ADMD und Dignitas. Der Verein Dignitas begleitete am häufigsten Suizidtouristen, es folgt der Verein Exit International, dann die anderen Vereine. Zudem scheint es einen Zusammenhang zu geben zwischen den einzelnen Schweizer Regionen und den dort aktiven Suizidbeihilfeorganisationen. Unsere Analyse zeigte dies ausdrücklich für das französische Gebiet der Schweiz, in dem fast alle AS-Fälle von $A D M D$ begleitet worden waren. Personen, die aus dem Ausland angereist sind, wurden im Kanton Zürich ausschliesslich von Dignitas und in der Schweizer Mittellandregion von EXIT International begleitet.

Bei den zugrundeliegenden Erkrankungen dominieren die Malignome (24\%) knapp vor den Erkrankungen aus dem neurologischen (17\%) und denen aus dem rheumatologischen (14\%) Formenkreis. Als häufigste Suizidmotive wurden körperliche Beschwerden wie dro- 
hender Verlust von Körperfunktionen und unzureichende Schmerzkontrolle gefolgt von Verlust der Lebensqualität genannt. ${ }^{4}$

\section{Spezifische Probleme}

Da der AS nach Artikel 253 Absatz 1 der Schweizerischen Strafprozessordnung (SR 312.0 StPO) als aussergewöhnlicher Todesfall (agT) behandelt wird, besteht eine Meldepflicht an die Strafermittlungsbehörden. In der Regel muss ein/e Arzt/Ärztin nach Feststellung des Todes die Behörden resp. die Polizei über den agT verständigen. Am Leichenfundort wird eine amtsärztliche Untersuchung (Legalinspektion) durchgeführt. Die Legalinspektion dient neben der Klärung der Todeszeit auch der Klärung der Todesart und Todesursache und der Identifizierung des Leichnams. Des Weiteren müssen auch immer die Inhalte der beigelegten Dokumente auf Plausibilität überprüft werden. Dazu zählen gemäss Richtlinien eine ärztliche Bestätigung der Diagnose und der Urteilsfähigkeit, sowie ein Rezept über das Suizidmittel, ein Zeitprotokoll über den Ablauf der geleisteten Suizidbeihilfe, die handschriftlich unterzeichnete Freitoderklärung, ein Mitgliederausweis und ein Kostenblatt mit Auflistung der erbrachten Leistungen. Wenn sich im Rahmen der Legalinspektion keine Auffälligkeiten ergeben, wird der Leichnam durch die Staatsanwaltschaft freigegeben (Brunner et al. 2009).

Aufgrund der Legalinspektion sind Rechtsmedizinerinnen und Rechtsmediziner in der täglichen Arbeit immer mal wieder mit dem Problemfeld AS konfrontiert. Teil des Problemfelds ist die Todes- und Todeszeitfeststellung. Die Todesfeststellung wird als ärztliche Handlung betrachtet, mit welcher der Individualtod deklariert und damit jegliche weitere Behandlung ausgeschlossen wird. Die Feststellung des Todes erfolgt über die Prüfung der sicheren Todeszeichen wie Totenflecken und Totenstarre an der vollständig entkleideten Leiche. Können diese Todeszeichen von den eingetroffenen Ärzten nicht festgestellt werden, so muss eine Reanimierbarkeit vermutet werden und mit Reanimationsmassnahmen begonnen werden. Für diese Überprüfung braucht es also eine ärztliche Ausbildung, die die Suizidbegleiter in der Regel nicht haben. Dennoch nehmen diese die Todesfeststellung vor bzw. dokumentieren die Zeit des Todeseintritts in einem Protokoll. Eine solche Situation kann die Ärztin bzw. den Arzt in einen Konflikt bringen. Im Rahmen der Feststellung der Todeszeit ist es schon vorgekommen, dass die im Protokoll durch die Suizidbegleiter festgestellte Zeit nicht mit der ärztlich festgestellten übereingestimmt hat, wodurch es zu Friktionen bei der Todeszeitbestimmung kommt. Für die Ausstellung des Totenscheins und der Todesurkunde sollte bei der Leichenschau die Identitätsfeststellung erfolgen. Die Identitätsüberprüfung kann im Normalfall durch die Ausweisdokumente erfolgen. Die Einzelfallanalyse hat gezeigt, dass dies auch nicht immer einfach war. Besonders in den Fällen, in denen es zu alters- oder krankheitsbedingten deutlichen äusserlichen Veränderungen der Person gekommen war, konnte die Identität durch einen Abgleich mit dem Foto aus dem Ausweis nicht mehr sicher bestimmt werden (Bartsch 2016).

\footnotetext{
${ }^{4}$ Detailliertere Ergebnisse werden in einer folgenden Publikation beschrieben.
} 
Bei der Feststellung von Todesart und Todesursache können in AS-Fällen ebenfalls Probleme auftreten. Bei der Todesart geht es darum festzustellen, ob der Todeseintritt durch Fremdverschulden verursacht wurde. Im Rahmen eines AS muss also sichergestellt werden, dass die Einnahme des tödlichen Medikaments selbständig und ohne Unterstützung durch Dritte erfolgt ist. Je nach vorliegender krankheitsbedingter körperlicher Funktionseinschränkung kann es sein, dass eine orale Einnahme nicht möglich war. In einzelnen Fällen hat man selbstgebastelte Hilfsmittel der Suizidbeihilfeorganisationen gefunden, die eine Beurteilung, ob eine selbständige Aufnahme erfolgt ist, nicht zuliessen. Neben der Todesart muss aber auch die Todesursache, also der Auslöser für den Todeseintritt, festgestellt werden, was im Falle einer Intoxikation vor Ort nicht möglich ist, da von aussen keine Zeichen am Leichnam auf eine Vergiftung hinweisen. In der Regel wird bei einer vermuteten Intoxikation eine Obduktion inklusive chemisch-toxikologischer Analyse staatsanwaltschaftlich in Auftrag gegeben. Dies würde sich für eine weiter steigende Anzahl der AS nicht finanzieren lassen (Bartsch 2016).

Des Weiteren konnten die Rechtsmediziner im Rahmen der Dokumentenprüfung vor Ort widersprüchliche Angaben in den unterschiedlichen Dokumenten zu ein und demselben Patienten aufdecken. So gab eine Patientin in einem Bericht an, dass Schlucken problemlos möglich war und in einem anderen, dass durch die Erkrankung (Amyotrophe Lateralsklerose) Essen und Trinken beinahe unmöglich geworden waren. Besonders problematisch sind unvollständige oder widersprüchliche Dokumente im Zusammenhang mit der Beurteilung der Urteilsfähigkeit. Im Rahmen der Legalinspektion sollte die Überprüfung und Bestätigung der Urteilsfähigkeit erfolgen, was retrospektiv sehr schwierig ist. So stand z.B. bei einer Person, die Patientin sei voll urteilsfähig hinsichtlich ihres Freitodwunsches, während in einem anderen zeitnahen Arztbericht die Urteilsfähigkeit als nicht gegeben angesehen wurde. Die Daten aus der Studie wie auch die Analyse der Einzelfälle haben gezeigt, dass die Urteilsfähigkeit in der Regel immer durch die gleichen Ärztinnen und Ärzte, die für die Suizidbeihilfeorganisationen beratend tätig sind, beurteilt wird. So kam es oft vor - besonders bei Suizidwilligen, die aus dem Ausland angereist sind -, dass diese Ärzte die Personen bei der Beurteilung zum ersten $\mathrm{Mal}$ gesehen haben. Auch dies sind Umstände, die zu Spekulationen verleiten können (Bartsch 2016).

Diese Fallbeispiele zeigen, dass es sich um schwierige Situationen handelt, für die bisher offizielle einheitliche Handlungsanweisungen fehlen. In vielen Bereichen können aufwendige Nachuntersuchungen unter anderem aus finanziellen Gründen nicht erfolgen, so dass sich die Strafverfolgungsbehörden in diesen Fällen auf die Angaben der Suizidbeihilfeorganisationen verlassen müssen.

\section{Zusammenfassung}

Unsere und die aktuellen BfS-Daten haben gezeigt, dass AS-Fälle im Zeitverlauf weiterhin stetig zunehmen. Die betroffene Klientel ist zahlenmässig zumeist weiblich, im Durchschnitt 73 Jahre alt, leidet am häufigsten an einem Malignom und gibt am häufigsten körperliche Beschwerden und unzureichend kontrollierbare Schmerzen als Motiv für den Sterbewunsch an. 
Aktive Sterbehilfe und Suizidbeihilfe wurden seit den 90er Jahren in immer mehr Ländern der Erde mittels Schaffung von spezifischen gesetzlichen Regelungen legalisiert. Während zum Beispiel in den Niederlanden auch die aktive Form von Sterbehilfe (Euthanasie) erlaubt wurde, war im US-Bundesstaat Oregon ausschliesslich der physician assisted suicide (PAS) legitimiert worden (Andorno 2013). In den letzten Jahren gab es auf Schweizer Bundesebene, zum Teil aber auch in den Kantonen, viele politische Vorstösse, um die Suizidbeihilfe genauer, gegebenenfalls strenger zu reglementieren (Bosshard 2012). Die skizzierten Problemfelder belegen anschaulich, dass es bisher in der Schweiz trotz vorhandener Regelungen keine standardisierte Vorgehensweise bei der organisierten Suizidbeihilfe gibt. Es kann somit nicht sichergestellt werden, dass genügend Sorgfalt bei der Beurteilung und Ausführung angewendet wird. Durch die liberale Rechtslage können sich Suizidbeihilfeorganisationen innerhalb des rechtlichen Rahmens völlig autonom organisieren (Nationale Ethikkommission im Bereich der Humanmedizin 2006) und ihre Tätigkeit ohne die Bereitschaft zur Kooperation ausüben. In der medialen Berichterstattung hat vor allem die in der Schweiz weltweit einmalige Praxis der organisierten Suizidbeihilfe für im Ausland wohnhafte Personen für Aufsehen gesorgt, was zum häufig verwendeten Begriff des Sterbe- bzw. Suizidtourismus geführt hat (Gauthier et al. 2015). Seit der Gründung des Vereins Dignitas im Jahr 1998 wächst die Zahl der im Ausland ansässigen Suizidnachfragenden an, wobei der Verlauf vergleichbar ist mit den AS-Fällen der in der Schweiz ansässigen Personen. Insbesondere bei Fällen aus dem Ausland stellt sich die Frage, wie sorgfältig die Abklärung der Voraussetzungen für die Suizidbeihilfe erfolgt. Schwarzenegger et al. (2010) empfehlen für Suizidwillige, die aus dem Ausland anreisen, die Einführung einer Pflicht zu einem längeren Aufenthalt in der Schweiz. Hier sollte man jedoch abwägen, was den Sterbewilligen in einer solchen Situation zugemutet werden kann und welches Ausmass man fordert.

Mit dem Bundesgerichtsentscheid im Jahr 2006 wurde die ärztliche Verantwortung im Rahmen von Suizidbegleitungen stärker betont, indem insbesondere die Verschreibung des tödlichen Medikaments und die Beurteilung der Urteilsfähigkeit strenger reglementiert wurden (Bosshard 2012). Hingegen lehnte der Bundesrat die Idee ab, die Suizidbeihilfeorganisationen durch ein Aufsichtsgesetz zu kontrollieren, da eine daraus resultierende Institutionalisierung dieses Arbeitsfeldes unverhältnismässig sei (Bundesrat 2011).

Unsere Analysen konnten zeigen, dass das Fehlen von standardisiertem Vorgehen beim AS in der Schweiz zu Problemfällen führen kann. Um solche Problemfälle zu verringern, empfiehlt sich die Errichtung einer interdisziplinären Expertenkommission, welche aus Vertreterinnen und Vertretern von Suizidbeihilfeorganisationen, Forschung, Justiz und Medizin besteht. Diese Kommission sollte dann die Bedingungen für eine Zulassung zum AS sowie auch mögliche Sanktionen im Falle einer nicht sorgfältigen Überprüfung der Bedingungen festlegen. So könnten die Fälle unter Berücksichtigung dieser Bedingungen vor dem AS geprüft und entschieden werden und gleichzeitig würde sich eine postmortale Abklärung erübrigen. 


\section{Literaturverzeichnis}

Andorno, Roberto (2013). Nonphysician-assisted suicide in Switzerland. Cambridge Quarterly of Healthcare Ethics, 22(03), 246-253.

Bartsch, Christine (2016). Praxis und Probleme des assistierten Suizids in der Schweiz aus rechtsmedizinischer Sicht. In: Caroline Welsh, Christoph Ostgathe, Andreas Frewer und Heiner Bielefeldt (Hg.) (2011). Autonomie und Menschenrechte am Lebensende (203-220). Bielefeld: Transcript Verlag.

Bundesrat (2011). Palliative Care, Suizidprävention und organisierte Suizidhilfe. Bericht des Bundesrates, Bern, Bundesrat.

Bundesamt für Statistik (BfS) (2016). Statistik Schweiz - Todesursachenstatistik 2014: Assistierter Suizid (Sterbehilfe) und Suizid in der Schweiz. Internet-Seite. Zugriff am 14.11.16 auf https://www.bfs.admin.ch/bfs/de/home/statistiken/gesundheit/gesundheitszustand/sterblichkeittodesursachen/spezifische.assetdetail.1023131.html

Bosshard, Georg (2012). Beihilfe zum Suizid-medizinische, rechtliche und ethische Aspekte. Praxis, 101(3), 183-189.

Brunner, Andreas (2010). Graubereiche in der Sterbehilfe. In: Frank Th. Petermann (Hg.) (2010). Sterbehilfe im Fokus der Gesetzgebung (223-248). St. Gallen: Institut für Rechtswissenschaft.

Brunner, Andreas, Hans Wehrli und Ernst Haegi (2009). Vereinbarung über die organisierte Suizidhilfe. Internet-seite. Zugriff am 20.11.16 auf http://static.nzz.ch/files/4/7/6/EXIT-Vereinbarung2_1.2980476.pdf

DIGNITAS (2016). DIGNITAS - Menschenwürdig leben - Menschenwürdig sterben. Internet-Seite. Zugriff am 15.11.16 auf http://www.dignitas.ch/

EXIT Deutsche Schweiz (2016). EXIT auf einen Blick. Internetseite. Zugriff am 15.11 .16 auf https://www.exit.ch/exit-auf-einen-blick/.

Gauthier, Saskia, Julian Mausbach, Thomas Reisch und Christine Bartsch (2014). Suicide tourism: a pilot study on the Swiss phenomenon. J Med Ethics, 41, 611-617.

Mathwig, Frank (2010). Leben dürfen - Sterben können. Zur aktuellen Diskussion um die Suizidhilfe. Internet-Seite. Zugriff am15.12.16 auf http://www.kirchenbund.ch/sites/default/files/media/pdf/themen/sterbehilfe/leben-k-nnen-lebend-rfen.pdf

Nationale Ethikkommission im Bereich Humanmedizin (NEK) (2006). Sorgfaltskriterien im Umgang mit Suizidbeihilfe. NEK Bericht. Bern: Nationale Ethikkommission im Bereich Humanmedizin.

Schwarzenegger, Christian, Patrik Manzoni, Daniel Studer und Catia Leanza (2010). Was die Schweizer Bevölkerung von Sterbehilfe und Suizidbeihilfe hält. Erste Resultate einer repräsentativen nationalen Befragung. Jusletter. 13(9), 1-12.

Schweizerische Akademie der Medizinischen Wissenschaften (SAMW) (2013). Betreuung von Patientinnen und Patienten am Lebensende - Medizinisch ethische Richtlinien der SAMW. Internet-Seite. Zugriff am 15.11 .16 auf http://www.samw.ch/de/Ethik/Richtlinien/Aktuell-gueltige-Richtlinien.html.

Schweizerisches Bundesgericht (BGE) (2006a). Entscheide 2A.48/2006. 
- (2006b). Entscheide 2A.66/2006.

Schweizerisches Strafgesetzbuch vom 21. Dezember 1937 (Stand am 01. Januar 2015). Internet-Seite. Zugriff am 14.11.16 auf http://www.admin.ch/opc/de/classified-compilation/19370083/index.html.

Schweizerische Strafprozessordnung vom 05. Oktober 2007 (Stand am 01. Januar 2015). Internet-Seite. Zugriff am 14.11.16 auf http://www.admin.ch/opc/de/classified-compilation/20052319/index.html.

Schweizerisches Zivilgesetzbuch vom 10. September 1907 (Stand am 01. Juli 2013). Internetsite. Zugriff am 14.11.16 auf http://www.admin.ch/opc/de/classified-compilation/19070042/201307010000/210.pdf.

Trachsel, Manuel, Hermann, Helena und Biller-Andorno Nikola (2014). Urteilsfähigkeit: Ethische Relevanz, konzeptuelle Herausforderung und ärztliche Beurteilung. Swiss Medical Forum, 14, 221225 .

Van der Heide, Agnes, Luc Deliens, Karin Faisst, Tore Nilstun, Michael Norup, Eugenio Paci, Gerrit van der Wal und Paul J van der Maas (2003). End-of-life decision-making in six European countries: descriptive study. The Lancet, 362(9381), 345-350. 